\title{
THE REACTIVITY OF AROMATIC AND HETEROCYCLIC DERIVATIVES OF HYDRAZINE. VII. THE ACID-BASE PROPERTIES OF SUBSTITUTED 5,7-DICHLORO-9-HYDRAZINE ACRIDINE
}

\author{
A.O.Devyatkina, O.M.Svechnikova, S.V.Kolisnyk, N.P.Kobzar, O.F.Vinnik \\ National University of Pharmacy \\ Kharkiv National Pedagogical University named after G.S.Skovoroda
}

Key words: substituted 5,7-dichloro-9-hydrazine acridine; reactivity; Hammet correlation equation

\begin{abstract}
The reactivity of substituted 5,7-dichloro-9-hydrazine acridine that exhibit various pharmacological activity by studying acid-base equilibria in the ethanol-water binary solvent (50 Mol \% ethanol) at $25^{\circ} \mathrm{C}$ using the method of potentiometric titration has been investigated. The values $p K$ of the corresponding conjugate acids obtained for 7 compounds indicate that these compounds are very weak bases. Analysis of the impact of the nature and position of substituents in the heterocycle on the strength of the corresponding conjugate acids has shown that the acceptor substituents weaken basicity of 5,7-dichloro-9-hydrazine acridine, and donor substituents cause the opposite effect. The quantitative assessment of the influence of substituents in the molecule by the Hammett equation within the principle of linearity of free energies with convincing statistical parameters has shown a low sensitivity of the reaction centre to structural changes in the molecule of 5,7-dichloro-9-hydrazine acridine. Using the correlation equation and the value $\mathrm{pK}_{\mathrm{BH}}$ experimentally obtained for 1- $\mathrm{CH}_{3}$ substituent the $\sigma$-constant of this substituent: $\sigma\left(1-\mathrm{CH}_{3}\right)=0.056$ has been determined. The Hammett correlation equation is used to predict the acid-base properties of substituted 5,7-dichloro-9-hydrazine acridines and the molecular design of more active pharmacophores.
\end{abstract}

Substituted 9-hydrazine acridines exhibit the antimicrobial, antihypoxic, analgesic activity [3, 5, 6, 9-11] and are precursors for the synthesis of their various derivatives with new pharmacological properties. Therefore, the study of their reactivity is of undoubted scientific and practical interest since it allows optimizing the ways of their synthesis and predicting the biological activity.

\section{Materials and Methods}

The synthesis of substituted 5,7-dichloro-9-hydrazine acridine (1-7) was carried out according to the method [11], their physicochemical parameters are shown in Table.

The ionization constants of the compounds under research were determined by the method of potentiometric titration $[1,7,17]$ in the ethanol-water binary solvent $\left(50 \mathrm{Mol} \%\right.$ ethanol) at $25^{\circ} \mathrm{C}$. These constants are given in Table.

The study of acid-base equilibria was carried out by the method [8]. The titrant was $0.01 \mathrm{M}$ aqueous solution of $\mathrm{HCl}$. The concentration of the titrated solutions was $0.005 \mathrm{Mol} \cdot \mathrm{dm}^{-3}$ at the semineutralization point. Potentiometric titration was performed on an EB-74 ionomer with glass (ESP-43-074) and silver-silver chloride electrodes at $25^{\circ} \mathrm{C}$. The experiment was performed in triplicates and processed in accordance with the requirements of the SPhU [2]. The correlation analysis was conducted by the method of mathematical statistics (the confidence probability - 0.95) $[2,8]$.

To prepare the mixed solvent the $\mathrm{CO}_{2}$-free bidistillate and ethanol were used.

\section{Results and Discussion}

The reactivity of substituted 5,7-dichloro-9-hydrazine acridine of this isostructural series was studied in reversible conditions. Ionization of $\mathrm{NH}$-acids conjugated with substituted 5,7-dichloro-9-hydrazine acridine was studied according to the equation (Scheme).

The preliminary experiments have proven that on the $\mathrm{pH}-$ $\mathrm{f}\left(\mathrm{V}_{\mathrm{HC}}\right)$ plot there is only one point of intersection that coincides with the literature data $[4,12-15]$ about protonation of 9-hydrazine acridine only on the heterocyclic nitrogen.

The data in Table indicate that the compounds studied are very weak bases in contrast to substituted 9-aminoacridines [12]. This suggests the lack of resonance stabilization of the cation of 9-hydrazine acridine due to the isolating effect of the imino group. The introduction of acceptor substituents increases the positive charge at the reaction centre, therefore, the strength of conjugate acids with the acceptor substituent $\left(2-\mathrm{NO}_{2}, 4-\mathrm{NO}_{2}\right)$ regularly increases compared to the unsubstituted acid (the strength of the corresponding base reduces). Donor substituents cause the opposite effect.

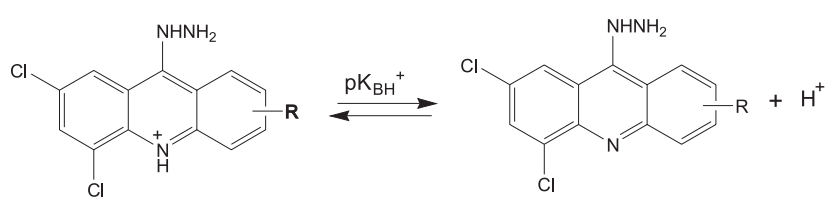
where: $\mathrm{R}=-\mathrm{H}, 1-\mathrm{CH}_{3}, 2-\mathrm{CH}_{3}, 3-\mathrm{CH}_{3}, 4-\mathrm{CH}_{3}, 2-\mathrm{NO}_{2}, 4-\mathrm{NO}_{2}$. 
The properties of substituted 5,7-dichloro-9-hydrazine acridine<smiles>Nc1c2ccccc2nc2c(Cl)cc(Cl)cc12</smiles>

\begin{tabular}{|c|c|c|c|c|c|c|c|c|c|c|c|}
\hline \multirow{2}{*}{ 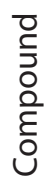 } & \multirow{2}{*}{$\mathrm{R}$} & \multirow{2}{*}{$\begin{array}{c}\text { Yield, } \\
\%\end{array}$} & \multirow{2}{*}{ M. p., ${ }^{\circ} \mathrm{C}$} & \multicolumn{3}{|c|}{ Found,\% } & \multirow{2}{*}{ Mol. formula } & \multicolumn{3}{|c|}{ Calc.,\% } & \multirow{2}{*}{$\mathrm{pK}_{\mathrm{BH}}{ }^{+}$} \\
\hline & & & & C & $\mathrm{N}$ & $\mathrm{H}$ & & C & $\mathrm{N}$ & $\mathrm{H}$ & \\
\hline 1 & $\mathrm{H}$ & 78 & $175-178$ & 56.37 & 15.18 & 2.92 & $\mathrm{C}_{13} \mathrm{H}_{8} \mathrm{Cl}_{2} \mathrm{~N}_{3}$ & 56.34 & 15.16 & 2.91 & $4.11 \pm 0.02$ \\
\hline 2 & $1-\mathrm{CH}_{3}$ & 75 & 190-193 & 57.53 & 14.40 & 3.77 & $\mathrm{C}_{14} \mathrm{H}_{11} \mathrm{Cl}_{2} \mathrm{~N}_{3}$ & 57.55 & 14.38 & 3.79 & $4.15 \pm 0.03$ \\
\hline 3 & $2-\mathrm{CH}_{3}$ & 77 & 204-205 & 57.58 & 14.41 & 3.80 & $\mathrm{C}_{14} \mathrm{H}_{11} \mathrm{Cl}_{2} \mathrm{~N}_{3}$ & 57.55 & 14.38 & 3.79 & $4.15 \pm 0.02$ \\
\hline 4 & $3-\mathrm{CH}_{3}$ & 74 & 214-217 & 57.59 & 14.36 & 3.78 & $\mathrm{C}_{14} \mathrm{H}_{11} \mathrm{Cl}_{2} \mathrm{~N}_{3}$ & 57.55 & 14.38 & 3.79 & $4.14 \pm 0.01$ \\
\hline 5 & $4-\mathrm{CH}_{3}$ & 75 & $185-188$ & 57.51 & 14.33 & 3.77 & $\mathrm{C}_{14} \mathrm{H}_{11} \mathrm{Cl}_{2} \mathrm{~N}_{3}$ & 57.55 & 14.38 & 3.79 & $4.13 \pm 0.01$ \\
\hline 6 & $2-\mathrm{NO}_{2}$ & 72 & $238-241$ & 48.29 & 17.38 & 2.50 & $\mathrm{C}_{13} \mathrm{H}_{8} \mathrm{Cl}_{2} \mathrm{~N}_{4} \mathrm{O}_{2}$ & 48.32 & 17.34 & 2.49 & $3.74 \pm 0.02$ \\
\hline 7 & $4-\mathrm{NO}_{2}$ & 73 & $220-223$ & 48.30 & 17.37 & 2.48 & $\mathrm{C}_{13} \mathrm{H}_{8} \mathrm{Cl}_{2} \mathrm{~N}_{4} \mathrm{O}_{2}$ & 48.32 & 17.34 & 2.49 & $3.79 \pm 0.02$ \\
\hline
\end{tabular}

The quantitative assessment of the influence of substituents in the molecule of 5,7-dichloro-9-hydrazine acridine was carried out by the Hammett equation within the principle of linearity of free energies.

Correlation occurred with all data except $\mathrm{pK}_{\mathrm{BH}^{+}}\left(1-\mathrm{CH}_{3}\right)$. For this substituent the $\sigma$-constant is absent. The correlation equation obtained has reliable statistical parameters, indicating the reliability of prediction:

$$
\begin{aligned}
& \mathrm{pK}_{\mathrm{BH}^{+}}=(4.11 \pm 0.04)-(0.72 \pm 0.01) \cdot \sigma \\
& \mathrm{n}=6 \quad \mathrm{r}=0.998 \quad \mathrm{~s}=0.054 .
\end{aligned}
$$

The reaction constant $\rho$ in the equation is small by the absolute value $(\rho=0.72)$, and it indicates the low sensitivity of the reaction centre to structural changes in the molecule of 5,7-dichloro-9-hydrazine acridine.

The plot of $\mathrm{pK}_{\mathrm{BH}^{+}}-\mathrm{f}(\sigma)$ is linear (Fig.)

Using the correlation equation and the value $\mathrm{pK}_{\mathrm{BH}^{+}}$ experimentally obtained for $1-\mathrm{CH}_{3}$ substituent the $\sigma$-constant of this substituent (Table) $-\sigma\left(1-\mathrm{CH}_{3}\right)=0.056$ was determined.

It is interesting to note that the reaction constant $\rho$ for substituted 5,7-dichloro-9-hydrazine acridine within the experimental error coincides with $\rho$ for substituted 9-aminoacridine [16], it indicates a single mechanism of electronic effects of substituents on the reaction centre.

CONCLUSIONS

1. The reactivity of substituted 5,7-dichloro-9-hydrazine acridine has been studied in reversible conditions by

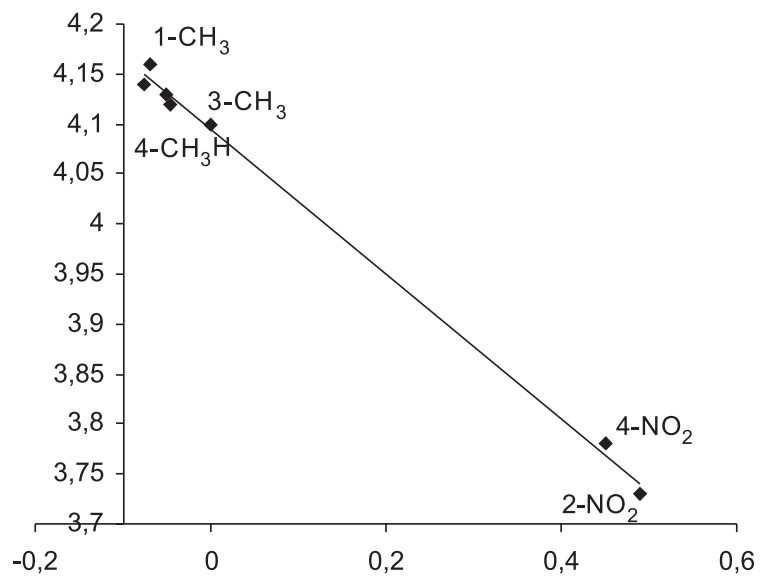

Fig. The plot of $\mathrm{pK}_{\mathrm{BH}^{+}}-\mathrm{f}(\sigma)$ for substituted 5,7-dichloro-9-hydrazine acridine.

investigating acid-base equilibria of conjugate acids in the mixed ethanol-water solvent $\left(50 \mathrm{Mol} \%\right.$ ethanol) at $25^{\circ} \mathrm{C}$.

2 . The impact of the nature and position of substituents in the heterocycle on the strength of the corresponding conjugate acids has been analyzed.

3 . It has been proven that the acceptor substituents weaken basicity of 5,7-dichloro-9-hydrazine acridine, and donor substituents cause the opposite effect.

4. The Hammett correlation equation with convincing statistical characteristics has been determined, it is used to predict the acid-base properties of substituted 5,7-dichloro-9-hydrazine acridines.

\section{REFERENCES}

1. Альберт А., Сержент Е. Константы ионизации кислот и оснований. - М.: Химия, 1964. - 178 с.

2. Державна фармакопея України / Державне підприємство «Науково-експертний фармакопейний иентр». 1-е вид. - Х.: РІРЕГ, 2001. - 556 c.

3. Ісаєв С.Г. // Ліки. - 2001. - №3/4. - С. 72-74.

4. саєв С.Г., Свєчнікова О.М., Павлій О.І. // Вісник фармації. - 2003. - №4 (36). - С. 27-29.

5. Ісаєв С.Г. // Фармац. журн. - 1999. - №3. - С. 52-54. 
6. Ісаєв С.Г., Яременко В.Д., Русакова Н.П. // Фармац. журн. - 2000. - №1. - С. 72-75.

7. Лабораторное руководство по хроматографии и смежным методам / Пер. с англ. - М.: Мир, 1982. T. 1. $-396 \mathrm{c}$.

8. Львовский Е.Н. Статистические методы построения эмпирических формул. - М.: Высш. шк., $1988 .-125$ с.

9. Мартиновський О.О., Панасенко Т.В., Панасенко О.І. // Вісник фармації. - 1999. - №2 (20). - C. 44-46.

10. Мохамед Мохамед Мохмуд Барака. Синтез, физико-химические свойства и биологическая активность тио-, гидразинопроизводных 6-хлор-7-нитроакридина: Автореф. дис. ... канд. фармац. наук. -X., 1992. - 19 с.

11. Панасенко А.Н., Омельянчик Л.А., Панасенко Т.В. та ін. // Актуальні питання фармацевтичної та медичної науки та практики. - Запоріжжя, 1988. - Т. 2, вип. 2. - С. 60-63.

12. Свечникова Е.Н. // ЖОХ. - 1997. - T. 67, выл. 1. - C. 138-140.

13. Свечникова Е.Н. // ЖОХ. - 1998. - T. 67, вып. 6. - С. 1007-1008.

14. Свечникова Е.Н. // ЖОХ. - 1999. - T. 68, вылn. 5. - С. 865-866.

15. Свечникова Е.Н. // ЖОХ. - 2001. - T. 71, выл. 5. - C. 848-849.

16. Свєчнікова О.М. Реакиійна здатність, зв'язок структура-біологічна активність та використання похідних N-фенілантранілової кислоти та акридину: Автореф. дис. ... докт. хім. наук. - Х., 1999. - 35 с.

17. Таблийы констант скорости и равновесия гетеролитических органических реакций. - М.: ВИНИТИ, 1975. - T. 1. -396 c.

РЕАКЦІЙНА ЗДАТНІСТЬ АРОМАТИЧНИХ ТА ГЕТЕРОЦИКЛІЧНИХ ПОХІДНИХ ГІДРАЗИНУ. VII. КИСЛОТНО-ОСНОВНІ ВЛАСТИВОСТІ ЗАМІЩЕНИХ 5,7-ДИХЛОРО-9-ГІДРАЗИНОАКРИДИНУ А.О.Девяткіна, О.М.Свєчнікова, С.В.Колісник, Н.П.Кобзар, О.Ф.Вінник

Ключові слова: заміщені 5,7-дихлоро-9-гідразиноакридину; реакційна здатність; кореляційне рівняння Гаммета

Досліджена реакційна здатність заміщених 5,7-дихлоро-9-гідразиноакридину, що проявляють різноманітну фрармакологічну активність, шляхом вивчення кислотно-основних рівноваг у бінарному розчиннику етанол-вода (50 мольних \% етанолу) при $25^{\circ} \mathrm{C}$ методом потенціометричного титрування. Одержані значення рК відповідних спряжених кислот для 7 сполук свідчать, що ці сполуки є вельми слабкими основами. Аналіз впливу природи і положення замісників у гетероциклі на силу відповідних спряжених кислот показав, що акцепторні замісники послаблюють основність 5,7-дихлоро-9-гідразиноакридину, а донорні чинять протилежний вплив. Кількісна оцінка впливу замісників у молекулі за рівнянням Гаммета у межах принципу лінійності вільних енергій з переконливими статистичними параметрами показала низьку чутливість реакційного центру до структурних змін у молекулі 5,7-дихлоро-9-гідразиноакридину. З використанням одержаного кореляційного рівняння і експериментально одержаного значення $\mathrm{K}_{\mathrm{BH}}$ для 1- $\mathrm{CH}_{3}$ заміщеного визначена б-константа цього замісника: $\sigma\left(1-\mathrm{CH}_{3}\right)=0,056$. Кореляційне рівняння Гаммета використовується для прогнозування кислотно-основних властивостей заміщених 5, 7-дихлоро-9-гідразиноакридинів та молекулярного дизайну більш активних фрармакофорів.

\section{РЕАКЦИОННАЯ СПОСОБНОСТЬ АРОМАТИЧЕСКИХ И ГЕТЕРОЦИКЛИЧЕСКИХ ПРОИЗВОДНЫХ ГИДРАЗИНА. VII. КИСЛОТНО-ОСНОВНЫЕ СВОЙСТВА ЗАМЕЩЕННЫХ 5,7-ДИХЛОР-9-ГИДРАЗИНОАКРИДИНА}

А.А.Девяткина, Е.Н.Свечникова, С.В.Колесник, Н.П.Кобзарь, А.Ф.Винник

Ключевые слова: замещенные 5,7-дихлор-9-гидразиноакридина; реакционная способность; корреляционное уравнение Гаммета

Исследована реакционная способность замещенных 5,7-дихлор-9-гидразиноакридина, проявляющих разнообразную фрармакологическую активность, путем изучения кислотно-основных равновесий в бинарном растворителе этанол-вода (50 мольных \% этанола) при $25^{\circ} \mathrm{C}$ методом потенциометрического титрования. Полученные значения рК соответствующих сопряженных кислот для 7 соединений свидетельствуют, что эти соединения являются слабыми основаниями. Анализ влияния природы и положення заместителей в гетероцикле на силу соответствующих сопряженных кислот показал, что акцепторные заместители ослабляют основность 5,7-дихлор-9-гидразиноакридина, а донорные оказывают противоположное влияние. Количественная оценка влияния заместителей в молекуле по уравнению Гаммета в рамках принципа линейности свободных энергий с убедительными статистическими параметрами показала низкую чувствительность реакционного центра к структурным изменениям в молекуле 5,7-дихлор-9-гидразиноакридина. С использованием полученного корреляци-

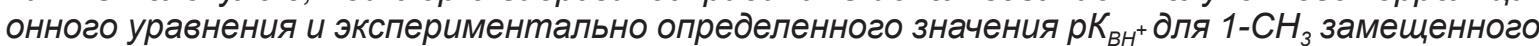
определена б-константа этого заместителя: $\sigma\left(1-\mathrm{CH}_{3}\right)=0,056$. Корреляционное уравнение Гаммета используется для прогнозирования кислотно-основных свойств замещенных 5,7-дихлор-9-гидразиноакридинов и молекулярного дизайна более активных фрармакофоров. 